\title{
Nutrient content of emergency food parcels provided during the COVID-19 pandemic in the UK
}

\author{
G. McNeill ${ }^{1}$ \\ ${ }^{1}$ Global Academy of Agriculture and Food Security, Royal (Dick) School of Veterinary Sciences, University of \\ Edinburgh, Edinburgh, UK
}

During the first lockdown of the COVID-19 pandemic in March 2020, UK local authorities arranged deliveries of emergency food parcels to those unable to access food supplies, with contents based on guidance from DEFRA ${ }^{(1)}$. The lunchtime food needs of the increased numbers of children entitled to free school meals were met through a grocery store voucher scheme worth $£ 15$ per child per week in England and by direct bank transfers of similar value in the devolved nations.

Schools closed again in January 2021 when food parcels intended to provide lunches for 5 days were distributed by local authorities in many areas in England. Images of parcel contents were posted on social media, one of which was described as 'mean' and 'depressing' by the mother ${ }^{(2)}$, leading to strong condemnation from food poverty campaigners ${ }^{(3)}$. Revised guidance on school lunch parcel contents was developed ${ }^{(4)}$ and the voucher scheme was revived.

This study aimed to compare the nutrient content of these parcels and of a hypothetical $£ 15$ basket of foods with recommended nutrient intakes.

Nutrient analysis of one emergency food parcel for adults; an initial school lunch parcel ${ }^{(2)}$; a modified school lunch parcel meeting the revised guidance ${ }^{(3)}$ and a hypothetical $£ 15$ basket of foods for children was carried out using Windiets 2017 . Results were compared with recommended nutrient intakes ${ }^{(5)}$ for adult women aged 19-50y or 14-16y old boys for energy, protein, fibre, iron, calcium and vitamins A, B1, B2, B3, B12, C and folate, apart from iron in 14-16y olds for which the recommendation for girls was used.

The emergency food parcel provided $95 \%$ of the energy, $85 \%$ of fibre and $80 \%$ of iron requirements and met all other daily nutrient recommendations for an adult woman aged 19-50y. The initial school lunch parcel provided $35 \%$ of energy requirements and over $30 \%$ of all other nutrient recommendations for an older child though salt content was $60 \%$ of the recommended daily intake. The parcel meeting the revised guidance provided $45 \%$ of energy requirements and higher intakes of all nutrients than the initial school lunch parcel, particularly of vitamins B2, B12, C and folate. The hypothetical $£ 15$ basket met $86 \%$ of the energy and all other daily nutrient recommendations for a 14-16y old child apart from salt (111\% of recommended).

All the food parcels met most nutritional recommendations, with the hypothetical $£ 15$ basket providing considerably more energy and certain nutrients than both the initial school lunch parcel and the parcel meeting the revised guidance. All parcels allowed few menu options and had limited scope for dietary choice or restrictions. Full examination of the strengths and weaknesses of these schemes could improve the design and delivery of similar schemes for the future.

\section{Acknowledgements}

I am grateful to Dr Kirsteen Shields, University of Edinburgh and Professor Elizabeth Dowler, University of Warwick, for their encouragement of and interest in this work.

\section{References}

1. https://www.gov.uk/government/news/over-1-million-food-boxes-delivered-to-those-most-at-risk-from-coronavirus

2. https://www.bbc.co.uk/news/explainers-53053337

3. https://www.bbc.co.uk/news/uk-55641740

4. https://laca.co.uk/laca-view/guidance-free-school-lunch-parcels

5. https://assets.publishing.service.gov.uk/government/uploads/system/uploads/attachment_data/file/618167/government_dietary_recommendations. pdf 